perform the combination procedure. These matters increase the cost, and the combination procedure would not be available in most institutions. Although EUS endoscopes have some features, such as a larger working channel (which enables the use of larger needles), wider ultrasonic scanning range, and adjustability of the protruding needle angle, the basic mechanism is similar to that of EBUS bronchoscopes. Use of the EBUS bronchoscope in place of the EUS endoscope might therefore be reasonable to simplify the procedure.

In conclusion, the use of the EBUS bronchoscope in place of the EUS endoscope for EUS-FNA in the diagnosis of mediastinal lesions is feasible in certain cases. This technique allows simpler mediastinal staging with high accuracy.

\section{References}

1. Rintoul RC, Skwarski KM, Murchison JT, Wallace WA, Walker WS, Penman ID. Endobronchial and endoscopic ultrasound-guided real-time fine-needle aspiration for mediastinal staging. Eur Respir J. 2005;25:416-21

2. Vilmann P, Krasnik M, Larsen SS, Jacobsen GK, Clementsen P. Transesophageal endoscopic ultrasound-guided fine-needle aspiration (EUS-FNA) and endobronchial ultrasound-guided transbronchial needle aspiration (EBUS-TBNA) biopsy: a combined approach in the evaluation of mediastinal lesions. Endoscopy. 2005; 37:833-9.

3. Wallace MB, Pascual JM, Raimondo M, Woodward TA, McComb BL, Crook JE, et al. Minimally invasive endoscopic staging of suspected lung cancer. JAMA. 2008;299:540-6.

4. Herth FJ, Eberhardt R, Krasnik M, Ernst A. Endoesophageal ultrasound-guided fine-needle aspiration (EUS-FNA) and endobronchial ultrasound-guided transbronchial needle aspiration (EBUS-TBNA) biopsy in the evaluation of mediastinal lesions using the EBUS-TBNA scope. Chest Meeting Abstracts. 2008;134:95003. Available at: http://meeting.chestpubs.org/cgi/content/abstract/134/4/p95003.

5. Janes SM, Spiro SG. Esophageal endoscopic ultrasound/endobronchial ultrasoundguided fine needle aspiration: a new dawn for the respiratory physician? Am J Respir Crit Care Med. 2007;175:297-9.

\title{
Subxiphoid epicardial left ventricular pacing lead placement is feasible
}

\author{
Takeyoshi Ota, MD, PhD, ${ }^{\text {a }}$ David Schwartzman, MD, ${ }^{\mathrm{b}}$ and Marco A. Zenati, MD, ${ }^{\mathrm{a}}$ Pittsburgh, Pa
}

\section{CLINICAL SUMMARY}

A 67-year-old woman, who had a longstanding history of symptomatic paroxysmal atrial fibrillation (AF) with rapid ventricular response intolerant of all pharmacotherapy, was referred for surgical epicardial lead placement for cardiac resynchronization therapy (CRT). Her medical history was significant for status-post left mastectomy for breast cancer, for which she was scheduled for left breast radiation therapy. Also, she had a right subclavian Mediport vascular access port (Cormed, Inc, Baltimore, Md) for chemotherapy. Since (1) the left infraclavicular area required radiation that precluded access for the transvenous lead placement on the left side, (2) the right subclavian Mediport access port also precluded access on the right side, and (3) irradiation would compromise wound healing of classic left thoracotomy approaches (eg, minithoracotomy, video-assisted thoracoscopy), we decided to perform the atrioventricular (AV)

\footnotetext{
From the Divisions of Cardiac Surgery ${ }^{\mathrm{a}}$ and Cardiology, ${ }^{\mathrm{b}}$ University of Pittsburgh, Pittsburgh, Pa.

Disclosures: None.

Received for publication May 24, 2009; revisions received June 10, 2009; accepted for publication June 10, 2009; available ahead of print Oct 9, 2009.

Address for reprints: Marco A. Zenati, MD, Professor, Division of Cardiac Surgery, University of Pittsburgh, C700 PUH, 200 Lothrop St, Pittsburgh, PA 15213 (E-mail: zenatim@upmc.edu).

J Thorac Cardiovasc Surg 2010;139:1661-2

$0022-5223 / \$ 36.00$

Copyright (c) 2010 by The American Association for Thoracic Surgery

doi:10.1016/j.jtcvs.2009.06.031
}

node ablation followed by epicardial lead placement through a subxiphoid approach.

After successful AV node ablation in the electrophysiology laboratory, a 1-inch subxiphoid incision was performed and the subxiphoid cartilage was excised. The sternum was retracted cranially with a subxiphoid retractor (Rultract; Rultract Inc, Cleveland, Ohio) (Figure 1). The pericardium was opened with stay sutures. By use of a heart positioner (Pyramid Positioner; ESTECH, San Ramon, Calif), the heart was elevated and the posterolateral wall was exposed without interfering with hemodynamic parameters (Figure 1). Bipolar epicardial steroid-eluting leads (CapSure Epi-model4968; Medtronic, Inc, Minneapolis, Minn) were implanted on the posterolateral wall of the left ventricle with 5-0 polypropylene sutures. Impedance of the left ventricular lead was $602 \mathrm{ohms}$ with a threshold of $2.5 \mathrm{~V}$. Additional ventricular leads (Medtronic) were placed on the right ventricle by the same technique (impedance $545 \mathrm{ohms}$ with a threshold of $2.5 \mathrm{~V}$ ), and a pacing device (InSync III model 8042; Medtronic) was connected to these leads and implanted in the right upper quadrant of the abdominal wall.

The postoperative course was uneventful with no complication. At 3 months' follow-up, both leads provided an adequate synchronization for CRT and satisfactory impedance and threshold (impedance 552 and 622 ohms with thresholds of $1.0 \mathrm{~V}$ and $1.0 \mathrm{~V}$ ), and the patient was doing well with episodes of sinus rhythm interspersed with AF. She was unaware of these transitions and was asymptomatic. 


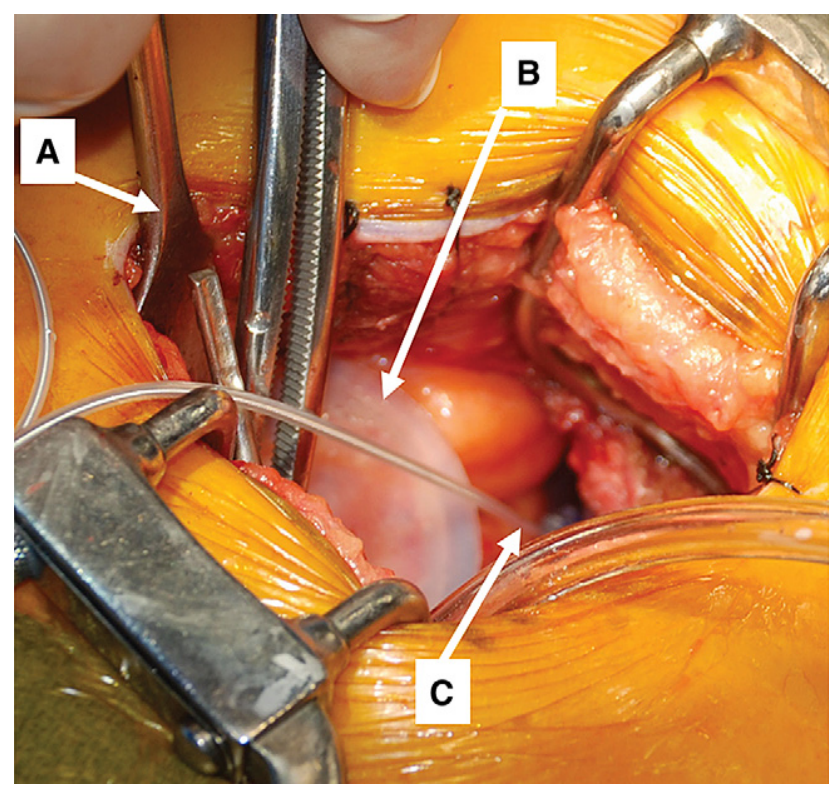

FIGURE 1. Intraoperative picture. The posterolateral wall of the left ventricle is exposed with a subxiphoid sternal retractor $(A)$ and a heart positioner $(B)$. An epicardial lead is implanted on the posterolateral wall $(C)$.

\section{DISCUSSION}

Percutaneous transvenous placement of left ventricular leads is currently the mainstay for CRT. The percutaneous procedure, however, can be challenging owing to a lack of operator experience, unsuitable coronary venous distribution for CRT, and significant amount usage of contrast medium and exposure to fluoroscopy. Its overall success rate is about $90 \%$ of attempts, ${ }^{1}$ whereas suboptimal lead positioning occurs in $30 \%$ of "successfully placed" left ventricular leads. ${ }^{2}$ The transvenous approach also involves major complications such as coronary sinus perforation and/or dissection, cardiac arrest, phrenic nerve stimulation, and lead dislodgment.

Given the limitations of percutaneous placement, surgical approaches are becoming important for CRT. Some minimally invasive approaches have been reported, such as videoassisted thoracoscopy, subxiphoid videopericardioscopy, ${ }^{3}$ and a robotically assisted approach. ${ }^{4} \mathrm{We}$ are interested in a sub- xiphoid approach, in which there are no significant anatomic barriers as well as no need for single-lung ventilation during surgery. In the present case, knowing that our strategy would lose the benefits from AV synchrony during the $30 \%$ of the time the patient is in sinus rhythm, we believed that consistent, drug-free ventricular rate control was critical given her very symptomatic AF refractory to any medical therapy. The patient was scheduled for radiation therapy on the left side, and the right subclavian vein was occupied for insertion of a Mediport vascular port for chemotherapy. Therefore, we believe that this patient was a good candidate for a subxiphoid epicardial left ventricular lead placement, considering that she had no access for a percutaneous approach. A controversial topic would be if we could place left ventricular leads in the right position. Placing left ventricular leads in adequate sites is important to achieving hemodynamic and functional benefits of CRT. The posterolateral wall of the left ventricle is a favorable site for left ventricular leads to get effective hemodynamic indices. A subxiphoid retractor and a heart positioner provided a proper operative field and direct visualization of the posterolateral wall of the left ventricle. Although this method may not be usable in patients with severe cardiomegaly, we believe the subxiphoid approach can be an alternative option for surgical epicardial left ventricular lead placement. In the future, advanced robotic technology would expand the indication of a subxiphoid approach for epicardial interventions, including epicardial lead placement for CRT. ${ }^{5}$

\section{References}

1. León AR, Abraham WT, Curtis AB, Daubert JP, Fisher WG, Gurley J, et al. MIRACLE Study Program. Safety of transvenous cardiac resynchronization system implantation in patients with chronic heart failure: combined results of over 2,000 patients from a multicenter study program. J Am Coll Cardiol. 2005;46:2348-56.

2. Mair H, Sachweh J, Meuris B, Nollert G, Schmoeckel M, Schuetz A, et al. Surgical epicardial left ventricular lead versus coronary sinus lead placement in biventricular pacing. Eur J Cardiothorac Surg. 2005;27:235-42.

3. Zenati MA, Bonanomi G, Chin AK, Schwartzman D. Left heart pacing lead implantation using subxiphoid videopericardioscopy. J Cardiovasc Electrophysiol. 2003;14:949-53.

4. Shalaby A, Sharma MS, Zenati MA. Robotic implantation of a multichamber cardiac resynchronization therapy defibrillator. Pacing Clin Electrophysiol. 2006;29: 906-9.

5. Ota T, Patronik NA, Schwartzman D, Riviere CN, Zenati MA. Minimally invasive epicardial injections using a novel semiautonomous robotic device. Circulation. 2008;118(14 Suppl):S115-20. 